\section{Plant banks miss crucial seeds}

Wild relatives of crop plants are largely missing from seed banks and plant repositories designed to protect biodiversity.

These relatives have genes that could be used to increase yields of crops or make them more resilient. Nora Castañeda-Álvarez at the International Center for Tropical Agriculture in Cali, Colombia, and her colleagues looked at 1,076 relatives of 81 crops, and found that only 45 relatives were adequately represented in seed banks. None of the 81 crops had its wild relatives sufficiently represented in such banks.

The authors call for systematic collecting of wild relatives, and highlight cassava, potato and sorghum as among the highest-priority crops.

Nature Plants http://dx.doi. org/10.1038/nplants.2016.22 (2016)

\section{ENGINEERING}

\section{Artificial eye sees in the dark}

Taking inspiration from the eyes of an unusual fish, researchers have created a device that can improve the ability of cameras to capture images in low light.

The elephantnose fish (Gnathonemus petersii), is known for its low-light vision, and its retina has many reflective microscopic cups that collect light. Hongrui Jiang and his colleagues at the University of Wisconsin-Madison made an array of microscopic cups from glass, lined with reflective aluminium. By transferring

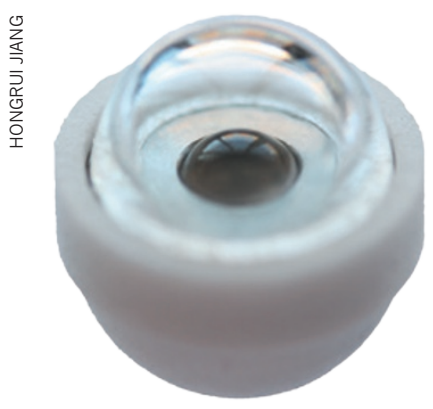

the cups to a stretched silicone polymer sheet, the authors shaped the array into a retinalike dome (pictured). The cups concentrated incoming light, boosting the sensitivity of the eye by more than three times compared to cameras that did not use this device.

The technique blurs the picture slightly, so the team applied an algorithm to sharpen it. The low-cost technique could enhance electronic sensors, which are reaching the limits of their sensitivity, and could have uses in night-vision robots, endoscopes and telescopes, say the authors.

Proc. Natl Acad. Sci. USA http://doi.org/bdhc (2016)

\section{GANCER BIOLOGY}

\section{Cancer cells get care packages}

Healthy cells that surround a tumour supply it with metabolites that support the voracious appetite of cancer cells - and could one day be targeted by therapeutics.

Cells can swap molecules by producing membranebound sacs called exosomes, which act as shuttles between cells. Deepak Nagrath of Rice University in Houston, Texas, and his team studied exosomes from normal connective-tissue cells associated with prostate and pancreatic cancers. They found that the activity of certain metabolic pathways increased in cancer cells when they took up exosomes from nearby normal cells.

The exosomes transported amino acids, lipids and other metabolites to the cancer cells. This cargo helped to sustain tumour growth when nutrients were limiting.

eLife http://dx.doi.org/10.7554/ elife.10250 (2016)

\section{BIOMECHANICS \\ Right prosthetic legs have the edge}

An athlete racing with a left-leg prosthesis might run more slowly than a competitor with a

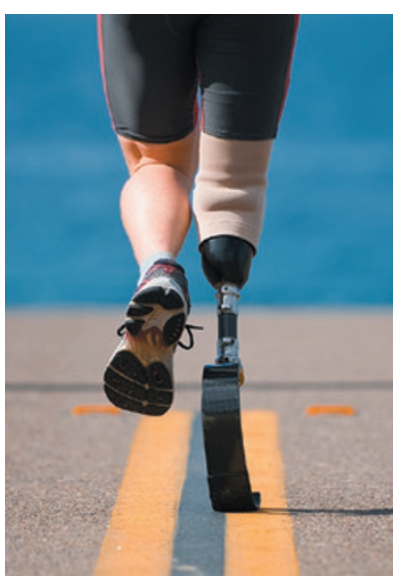

right-side prosthesis (pictured) at the Paralympic Games, because races are run in the anticlockwise direction.

The speed at which people run round a curve is thought to be limited by the forces exerted by the leg on the inside of the curve. To test this, Paolo Taboga of the University of Colorado Boulder and his team measured the running speeds of 11 athletes wearing leg prostheses. Those with one prosthetic leg were, on average, 3.9\% slower when their prosthetic leg was on the inside of the curve, compared with when it was on the outside.

All track events at the Paralympic Games are run on an anticlockwise track, and so athletes with rightleg prostheses may have an advantage over those with left-leg ones.

J. Exp. Biol. 219, 851-858 (2016)

\section{PLANETARY SCIENCE}

\section{Cassini aids hunt for Planet Nine}

Researchers using the Cassini spacecraft have narrowed down the search for the Solar System's hypothetical ninth planet.

Planet Nine is thought to be orbiting in the far outer Solar System, but has not yet been found. If it exists, its gravity should tug slightly on the outer planets, including Saturn. To determine Saturn's orbit, Agnès Fienga at the University of Nice Sophia Antipolis in Valbonne, France, and her colleagues used data from the ground-based radiodish network that tracks the position of Cassini, which has been orbiting Saturn since 2004. Using a Solar System model refitted with Planet Nine, the researchers conclude that if it exists, the planet probably lies along a particular $21^{\circ}$ slice of its predicted orbit.

If Cassini continues to operate until 2020, data from the spacecraft would further improve the estimate of the planet's location, the team says. Astron. Astrophys. 587, L8 (2016)

\section{NEUROSCIENCE}

\section{Forgetting inhibits new memories}

Suppressing unwanted memories could interfere with the creation of new ones.

Michael Anderson of the University of Cambridge, $\mathrm{UK}$, and his colleagues asked volunteers to learn pairs of words such as 'pump' and 'oil'. The participants were then cued by one word and asked to either recall, or purposely not think about, the other word in the pair. Between these trials, people viewed various scenes and had to imagine how an object came to be in the scene. The researchers found that, compared with no memory suppression, participants accurately recalled the object's identity about $45 \%$ less frequently if the scene was presented shortly before or after memory-suppression trials. The extent of the forgetting effect correlated with how much hippocampus activity was dampened during the memory-suppression trials. (The hippocampus is an area of the brain that is involved in memory processing.)

This 'amnesic shadow' could help to explain memory lapses that can follow traumatic experiences, when people try to suppress certain memories, the authors suggest.

Nature Commun. 7, 11003 (2016)

\section{ONATURE.COM}

For the latest research published by Naturevisit:

www.nature.com/latestresearch 